\title{
A pilot study of physician personal preferences for treatment of glioblastoma
}

\author{
Clark C. Chen ${ }^{1} \cdot$ Colin Depp $^{2} \cdot$ Bayard Wilson $^{3} \cdot$ Jiri Bartek Jr ${ }^{4,5,6} \cdot$ Bob Carter $^{1}$
}

Received: 21 July 2016/Accepted: 27 July 2016 / Published online: 19 August 2016

(C) Springer-Verlag Wien 2016

Dear Editor,

Most studies that examine the notion of gross total resection (GTR) in glioblastoma treatment are conducted with the assumption that extended survival is universally desirable [2]. There are limited data in terms of how such survival benefits should be weighed against the risk of the surgery and the impact of surgical morbidity on the patient's quality of life [1].

To study this issue, we designed a survey entitled "Putting yourself in your patient's shoes: a pilot study of physician personal preferences for treatment of glioblastoma" (U.C.S.D. institutional review board protocol no. 151821), where we survey physician members who have cared for glioblastoma patients. These physicians are well-acquainted with the consequences of surgery performed for glioblastoma located in different regions. We pose the question of whether the respondent would elect for GTR if s/he were afflicted with glioblastoma located in the right frontal lobe, right hemisphere, left hemisphere, or the posterior corpus callosum. Information on physician age, marital status, medical specialty (neurosurgery, neuro-oncology, medical oncology, neuroradi-

Jiri Bartek, Jr

jiri.bartek@karolinska.se

Department of Neurosurgery, UCSD, San Diego, USA

2 Department of Psychiatry, UCSD, San Diego, USA

3 UCSD School of Medicine, San Diego, USA

4 Department of Clinical Neuroscience, Section for Neurosurgery, Karolinska Institutet, Stockholm, Sweden

5 Department of Neurosurgery, Karolinska University Hospital, Stockholm, Sweden

6 Department of Neurosurgery, Copenhagen University Hospital Rigshospitalet, Copenhagen, Denmark ology, neuropathology or radiation oncology), years of practice, and personal values will be collected.

We would like to make neurosurgeons in Europe aware of this study, and to invite them to take part in it. We hope this study will give us more insight into our own preferences as physicans, when faced with the decision we council our patients on how to make on a daily basis.

To participate in the study please go to the following webpage by 31 October 2016: http://www.surveymonkey. com/r/Eu_preference_GBM.

\section{References}

1. Ausman JI (2014) Gross total resection: do we want survival statistics or quality of life measurements. Surg Neurol Int 5:77

2. Kramm CM, Wagner S, Van Gool S, Schmid H, Strater R, Gnekow A, Rutkowski S, Wolff JE (2006) Improved survival after gross total resection of malignant gliomas in pediatric patients from the HITGBM studies. Anticancer Res 26:3773-3779 